\title{
Design and Implementation of Web-based System for Client-based Design Project Management
}

\section{Dr. John P Puccinelli, University of Wisconsin, Madison}

Dr. Puccinelli is the Associate Chair of the Undergraduate Program in the Department of Biomedical Engineering. He began here as student near the start of the UW-BME program and earned his BS, MS, and $\mathrm{PhD}$ in BME. He is interested in hands-on instruction - teaching and developing courses related to biomaterials and tissue engineering, as well as design. He was awarded the BMES Student Chapter Teaching Award in 2011, 2013, and 2014 and the Polygon Outstanding BME Instructor Award in 2012.

\section{Mr. Matthew S Bollom}

Matthew Bollom is a 2013 graduate of the University of Wisconsin-Madison with a degree in Biomedical Engineering. He currently works for National Instruments in Austin, Texas. 


\section{Design and Implementation of Web-based System for Client-based Design Project Management}

\section{Introduction}

Biomedical engineering (BME) students at the University of Wisconsin-Madison participate in hands-on, client-based, real-world, team-based design projects for six semesters (sophomoresenior year). ${ }^{1,2}$ Through the design process, the students learn a variety of professional and technical skills. The design curriculum is advised by up to 16 faculty members per semester, each overseeing up to four teams. These design projects are chosen on the first day of class using a priority system developed by our Biomedical Student Advisory Committee (BSAC) and student teams are expected to maintain a project website to convey their progress and submit deliverables electronically. During our department's early years, the assignment process involved paper lists, sign-up sheets on walls, and manual counting to ensure that a sufficient number of projects were being assigned. Often one person would sign up for a project and then be forced to switch to a different (and possibly less desirable) project to ensure all teams were full. Following the completion of project selection, our Biomedical Web Implementation Group (BWIG) chair would manually create the framework for the project web pages. As our department has doubled in size from 25 projects per semester to now over 50, we found the process becoming lengthy and prone to mistakes.

In order to better manage the challenges associated with a larger department and the increased usage of technology in today's classrooms, we developed a web-based, database-driven system starting in early 2010 that allows for the management of the entire design course and provides the opportunity for students to be involved in the entire process. This system consists of five principal parts: 1. Ideas, is a portal to allow clients to submit project ideas, problem statements, budgets and contact information. 2. Project Selection, is a drag-and-drop 'draft style' interface that allows students to quickly choose projects or submit pre-proposals for a project prior to selection. It also ensure an appropriate number of teams are started based on the desired team sizes and it automatically creates project websites for each team. 3. Project Builder, is a tool used by one student on each team assigned the role of BWIG who maintains his or her team's project websites and submits deliverables electronically through this interface. 4. Course, is a self-generating semester course schedule, list of design course advisors (contact information and expertise), course resources and assignment guidelines, and displays the auto-generated project presentation schedule and 5. Administration, a faculty tool to manage the semester, access deliverables, and download preformatted student information for use in other University systems (such as course evaluations). The system also allows various levels of access for the public and a $\log$ in to a 'My Projects' interface for management by the students, clients, advisors and course coordinators. The system was first used in September 2010 and has continued to evolve to meet the needs of our design courses.

The system is powered using modern web technologies including HTML, CSS, PHP, and Javascript. Data is stored and protected in a MySQL database. The use of standard technologies has allowed for multiple seamless server transitions and upgrades to the codebase from a development website. Students, clients and faculty are also able to use their personal computers or devices at any time to access the system without the need to install specialized software. 
The system has facilitated communication between the students and their clients and advisors. It has drastically reduced the hours required to organize the projects and course, thus allowing our faculty to focus more on the educational process rather than the logistics of administering the design courses. As our department has continued to grow, the system has shown its ability to scale and help maintain the focus of the course on learning.

\section{BME Design - Web Course Manager}

To help create this course management system, we built on over a decade of experience in project administration and analysis of our procedures. In early 2010, we realized our approach of using paper sheets posted on the wall to assign design projects was not sustainable as we had exceeded our allotted classroom time of two hours when the assignment process was only about two-thirds done. This caused us to investigate alternatives that would allow us to accelerate the project assignment process and ensure our students had the opportunity to meet with their advisor on the first course day and learn what was expected of them over the course of the semester.

Our design faculty discussed this with our department's BSAC and gained their support. One of our students expressed interest in helping to develop this software and this software forms the framework of our current web course manager. At the time and still, there is not anything available commercially that would meet the needs of client-based design project selection and project management without drastically changing the selection process. ${ }^{3}$ The project initially started out as a means to address the growing pains associated with the project assignment process and the creation of the project webpages, but it quickly evolved to encapsulate nearly all of the design course management.

To understand what components and features would be necessary, we discussed the needs and concerns with students, design faculty, and clients. Through these conversations, we divided typical course activities into five sections protected by appropriate access levels: Ideas, Project Section, Project Builder, Course, and My Projects/Administration. Each of the five sections is highlighted below and each is designed for a specific subset of the system's users.

Users are divided into six separate categories:

1. Public - can only access publically available design project information and can submit project ideas to become a client

2. Guest - can be given access to specific protected design project information

3. Client - can access each of their current and previous design projects and can submit additional project ideas

4. Student - can access each of their current and previous design projects, submit project proposals to pre-select a project, participate in Selection, and access Project Builder

5. Advisor - the Design Faculty can access every project and information in Ideas and Selection (newly submitted projects)

6. Administrator - the course coordinator and BWIG chair can access and control all system features, including settings and Administration features and access all project information

Users and permissions are automatically created and assigned based on student rosters obtained from the University and the project information submitted from the client. Administrators have 
the ability to add new users and promote users to new access levels as necessary through the Administration features of the software.

All website links below will have the prefix http://bmedesign.engr.wisc.edu e.g.: .../ideas/

\section{Ideas}

Projects are continuously solicited from clients in order to allow students to choose from a bank of projects at the start of each semester. One mass email to clinicians, faculty, local companies, and non-profits in the summer and a smaller email to past clients over winter break facilitates this process. The email contains brief information about BME Design and the web location .../ideas/ where projects can be submitted. This form is also available at top of every page within the website and under "My Projects" for users. Within the email and on the submission form resides a link to frequently asked questions that describes the course format and expectations of being a client (primarily: course goals, meeting expectations, budget, and intellectual property (IP)). The submission form requires potential clients to provide information about the proposed problem: title, description, available materials and supplies, references, check boxes for skills the project might likely require related to the students' field of study and interests, budget, yes/no for potential IP where if 'yes' is selected the project is automatically locked to the public, the client's information (salutation, name, affiliation, phone, and email), and a place to add alternate contacts.

Once a project is submitted, there are three places where project ideas reside in the system. 'Project pool' is for these newly submitted projects, 'project selection' is the list of projects the students will ultimately choose from for a given semester and/or projects currently being worked on, and 'archived projects' is for all other projects submitted (projects that have been completed, never selected, or not appropriate for BME Design, but were not deleted). In all locations, the projects are displayed on the web as tiles containing the name, project short name, date submitted, client name and action buttons to move the project between the three areas and edit the description. Only administrators have the editing functionality and can drag and drop the projects to reorganize them. Organizing projects by class level or needed expertise facilitates selection. Advisors are also able to see the projects to assist in the vetting process.

After collecting projects for the semester (or at any time) the course coordinator can review all of the new projects in the project pool. For appropriate projects, the coordinator adds information to help the students choose the project including the short name separated by an underscore that ultimately becomes the project web address, engineering and medical specialties are filled in via autocomplete from the database, and typos are corrected. The Design Faculty then meet to review all of the projects for the semester and approve them by adding them to selection or reject them (delete or archive them). Projects are sometimes also pre-assigned to a course level (sophomore-senior) depending on the expected difficulty or left open for any level. Projects that are pre-assigned are treated as if a team from that level has selected it (it turns the appropriate color and it is labeled with the proper course number) so students from that level know they can select it.

\section{Project Selection}

Prior to the start of every semester, the course coordinator firsts clicks "prepare for semester" which automatically establishes the new semester name (i.e. Spring 2015) and appropriate course 
numbers for that semester, archives the previous semester's project web pages, prepares the selection process, and sets up the schedule with Thanksgiving/Spring Break (see Administration below). The coordinator then uploads the course roster that was downloaded from the University's system. To aid in identification of the students' class level within the system, an identifier is added to each roster (i.e. $2=$ sophomore, $3=$ junior, and $4=$ senior) and the rosters are combined into one file. The coordinator also updates the list of advisors/Design Faculty for the semester. When the project list is ready, the coordinator releases the project list to the students and enables the students to submit pre-proposals for projects by the appropriate deadline. The students can then navigate to .../selection/ to see the list of projects and submit their proposal.

Students are able to choose their project one of two ways: 1 . Submit a pre-proposal prior to the first day of class for a project on the list or 2. when their name comes up on the first day of class based on their level and randomly assigned order. The pre-proposal process was also established by our BSAC and was added into the software in 2013. Proposals require at least two students to work together and put together 500 words that describe how their expertise and interests are uniquely suited to solve the client's problem. Once all of the proposals are submitted, the Design Faculty review them to accept them, prioritize them, or reject them (Fig. 1). Once one is accepted, the system automatically marks those in competition with it as prioritized. Prioritized students go to the top of the random priority list to choose near the start of project selection, thus their efforts in submitting a proposal are not wasted. Proposals are rarely rejected (for example, if bogus text is submitted), but if so, they remain in the same random order. Once all of the proposals are reviewed and each one has been acted upon, the course coordinator can automatically email the teams the results and the students are then automatically assigned to the proper project.

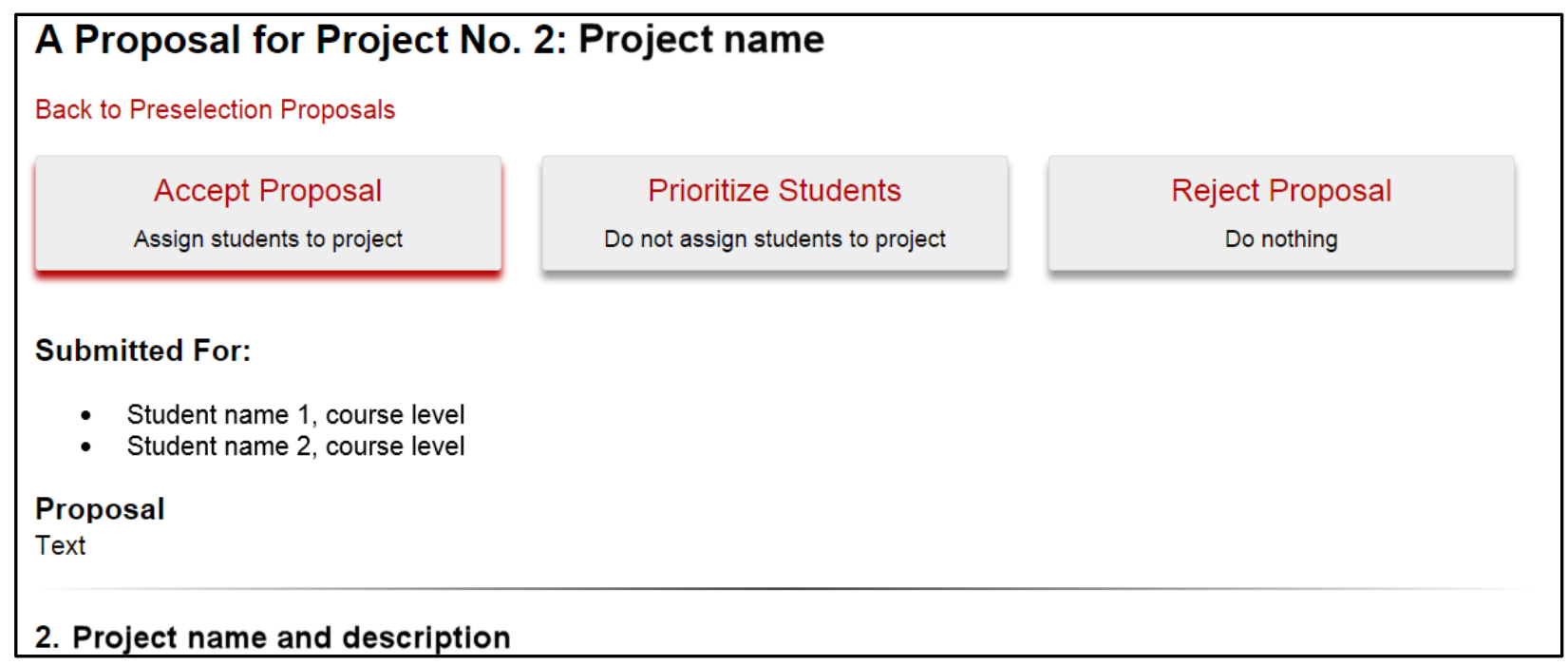

Fig. 1: Screenshot of a mock pre-proposal being reviewed by the Design Faculty. The project number, name, action buttons, students, proposal text and project description from the client are displayed. When there is more than one team that submitted a proposal for the same project then a next/previous button appears to facilitate the review. For this example, the course coordinator hit accept and thus it is highlighted. Once one team's proposal is accepted, all others for that project are automatically prioritized. 
With many students pre-assigned, the rest of the students will choose their project in real-time on the first day of class. The students already have access to the project list and descriptions from the pre-proposal process, as well as their order in the randomized priority list so they have an idea about what project(s) they want to work on. During selection, the web browser (Fig. 2) is displayed to the class full-screened showing all project numbers (going to the same website as the students .../selection/ the coordinator is able to assign the students while the students can only view the project information). The student choosing first (in the example, Megan Platner) says the project number desired ' 3 ' and the coordinator drags the name to the project. The next four people already know their names are coming up and are ready to select. In our process, students are also allowed to bring teammates with them into a project. To do this, the coordinator can type the teammate's name into the search box. As the name is being typed, suggestions populate the top bar. For example typing "Aly" would have brought up Mitchell, Alyssa or other relevant results. Any of the five names at the top can be dragged into a project.

Once a project is full with four people (as stipulated in "team set-up"), the project turns red. The number of students in a team is also indicated in the upper right of each project box. At the bottom of the selection page, the software also displays statistics and predictions for the remaining students and teams. Mousing over "team setup" shows how many four- and fiveperson teams are predicted and clicking allows for the team size to be adjusted. Once the proper number of teams have been started (i.e. Fig. 2 shows 15 teams to go), the rest of the projects that were unpicked disappear from selection and the remaining students must fill up the existing teams.

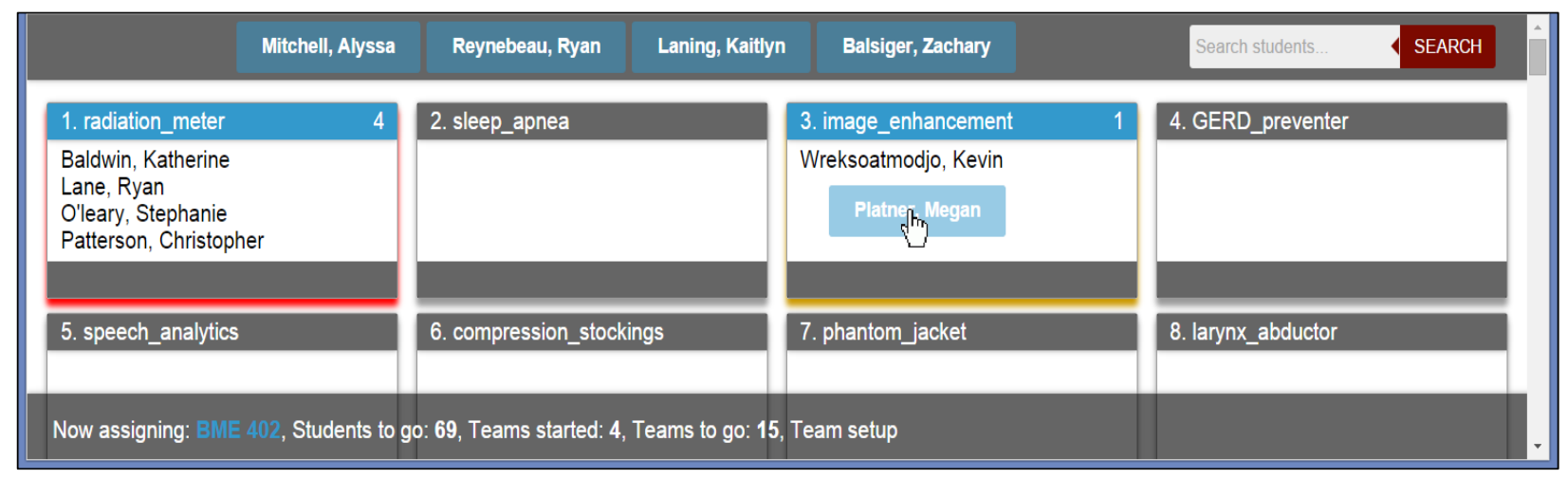

Fig. 2: Screenshot of the project selection draft from a web browser. The browser has been zoomed to only show the first eight projects. Normally, all projects are displayed at one time. The randomized students' names appear at the top of the screen showing the next five students to pick. The coordinator can then drag and drop the students based on the project they select. For example Megan is being dragged into project 3. In real-time, the projects fill (project 1 is red) and the number of students appears in the upper right of each project box. The bottom of the screen updates the number of students left for that class, the number of teams started, and the projected number of teams left to be started based on the settings for the team size. Each class has a different color to distinguish student class and project level. These settings can all be customized.

After all students have been assigned, then the Design Faculty choose which projects they want to advise. In the same way that the students pick their projects, the advisor's name is dragged into the desired project. Once all of the students and advisors are assigned, the classroom given to each advisor is displayed with the project number to facilitate the first meeting of the student teams with their advisor. The students then convene in this assigned classroom for the remainder 
of the class period and future weeks. At this point, the student teams decide on their team roles (Leader, Communicator, BWIG, BSAC, and BPAG (Biomedical Purchasing and Accounting Group)) and the BWIG builds their webpage by navigating to .../build/. The initial building of the webpage is also automated: it populates student, client and advisor information, as well as the initial project description.

\section{Project Builder}

Throughout the semester, the students update their web page with deliverables and images through an interface called Project Builder. Access is automatically granted to the team's BWIG, whose primary role is to ensure the website is up-to-date and that deliverables are submitted in a timely fashion. Teams are required to provide a team photo, weekly progress reports, brief weekly status update, their product design specification, preliminary and end of semester course reports and presentation files, and images of their final design.

Project Builder abstracts the process of creating and styling web pages from the students to ensure that the pages have a consistent look and maintain accessibility standards. The Project Builder interface allows the BWIG to provide the following details regarding their project: Project information, Project Status, Uploads, Contact information, Preliminary Presentation (to upload their mid-semester presentation file for fast access during presentations), Secure Project Page (to prevent public viewing and protect IP). Project Builder takes the information provided by the students and automatically builds the most recent version of the page using the site template as shown in Fig. 3. Help documentation is provided throughout the interface and various checks within the fields ensure proper and secure data entry.

Administrators have access to Project Builder for all project pages and it gives them the additional ability to adjust 'Users' linked to the project, including modifying team roles and adding guests. They are also able to 'Unsecure' any page. Protected project information includes documents and images which may contain IP; the project description, team photo, and contact information is always available. Teams with protected pages can display a generic description to the public and secure a more detailed description behind the login. Only the immediate team (students and client), specified guests, and advisors may access any protected information.

Additionally, the archive of all project information and deliverables serves as a tool to perform assessment for ABET accreditation. Each year the department's Assessment Committee reviews the work from the prior year for achievement of our student outcomes. ${ }^{4}$ The project pages serve as the content for that review. Modes of continuous improvement often take form within the design courses. 


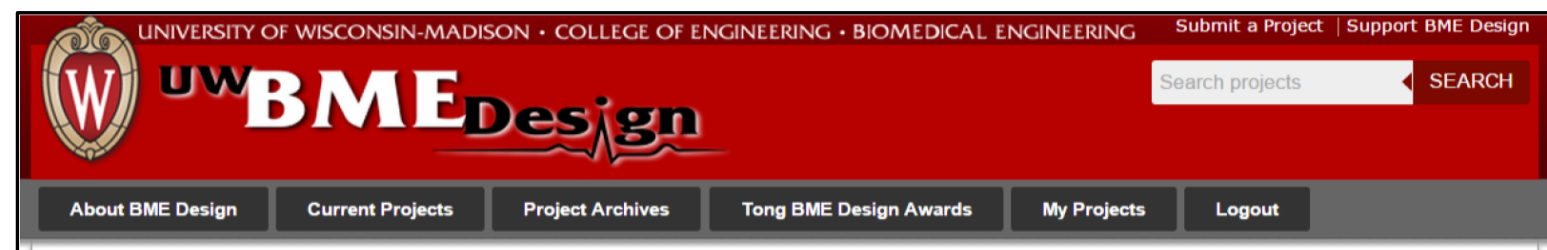

\section{Project name}

\section{Project Overview}

The problem statement and description as described by the students (initially as described the client).

\section{Project Status}

Weekly status updates are displayed here.

\section{Images}

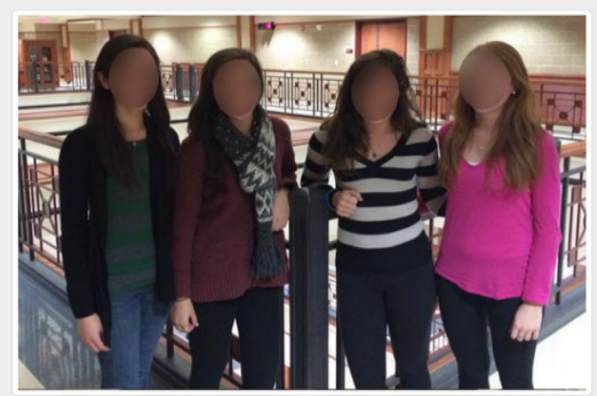

Team members from left to right: Member 1 Member 2, Member 3, Member 4

\section{Files}

Final deliverables (in pdf form) appear here as clickable tiles/links like the progress reports below.

\section{Progress reports}

February 21, 2014 Week 5
February 14, 2014 Week 4
February 7,2014 Week 3
January 24, 2014

Week 1

\section{Contact information}

\section{Team Members}

- Member 1 - Team Leader

- Member 2 - Communicator

- Member 3 - BSAC

- Member 4 - BWIG \& BPAG

Advisor and Client

- Prof. Advisor - Advisor

- Dr. Client - Client

Fig 3. Sample project page (note the affiliations from our University have been grayed out). The top header and navigation appear on all web pages except the draft-style selection process. This navigation is the same for all users except My Projects is only available when logged in. Progress reports are displayed as tiles and the dates are prefilled based on the semester schedule. Names appear as hyperlinks to email the specific individual. All progress reports and emails are removed from the public view from prior semesters. 


\section{Course}

Historically, we used another campus-available content management system (CMS) to host the design course guidelines and supporting documents. This included links to the project pages described above, PDFs of the course schedule, advisor contact information, instructions for all deliverables, links to design competitions, and resources to help with the design process, fabrication and testing. The course schedule provides an overview of what teams should aim to complete each week, by linking to the appropriate files and specified when deliverables were due. As the class size has been growing, maintaining advisor information for up to 16 faculty members that changes semester-to-semester in a web page html format became difficult. Additionally, the CMS employed changed the links every semester so the course schedule had to be re-built with each link requiring the updates.

To eliminate these complications, all course content was migrated into our own Design course manager system in 2014 where the "prepare for semester" updates all of the dates in the schedule (Fig. 4). Each file can individually be updated as necessary. Additionally, since the advisor's names were already stored for the selection process, additional information was added to include their contact information, link to their faculty profile, and their engineering expertise. The course page also contains a link to the presentation schedules for each group of advisors. This presentation schedule is automatically created based on the advisors and their assigned classrooms from the advisor interface, as well as the teams and desired presentation duration. The course coordinator is able adjust the schedule through drag and drop. When the semester starts, the students in the course have the 'course link' .../course/ appear under their "My projects" login to easily access course information. We also placed this link with minor text within the University's CMS for accessibility and availability.

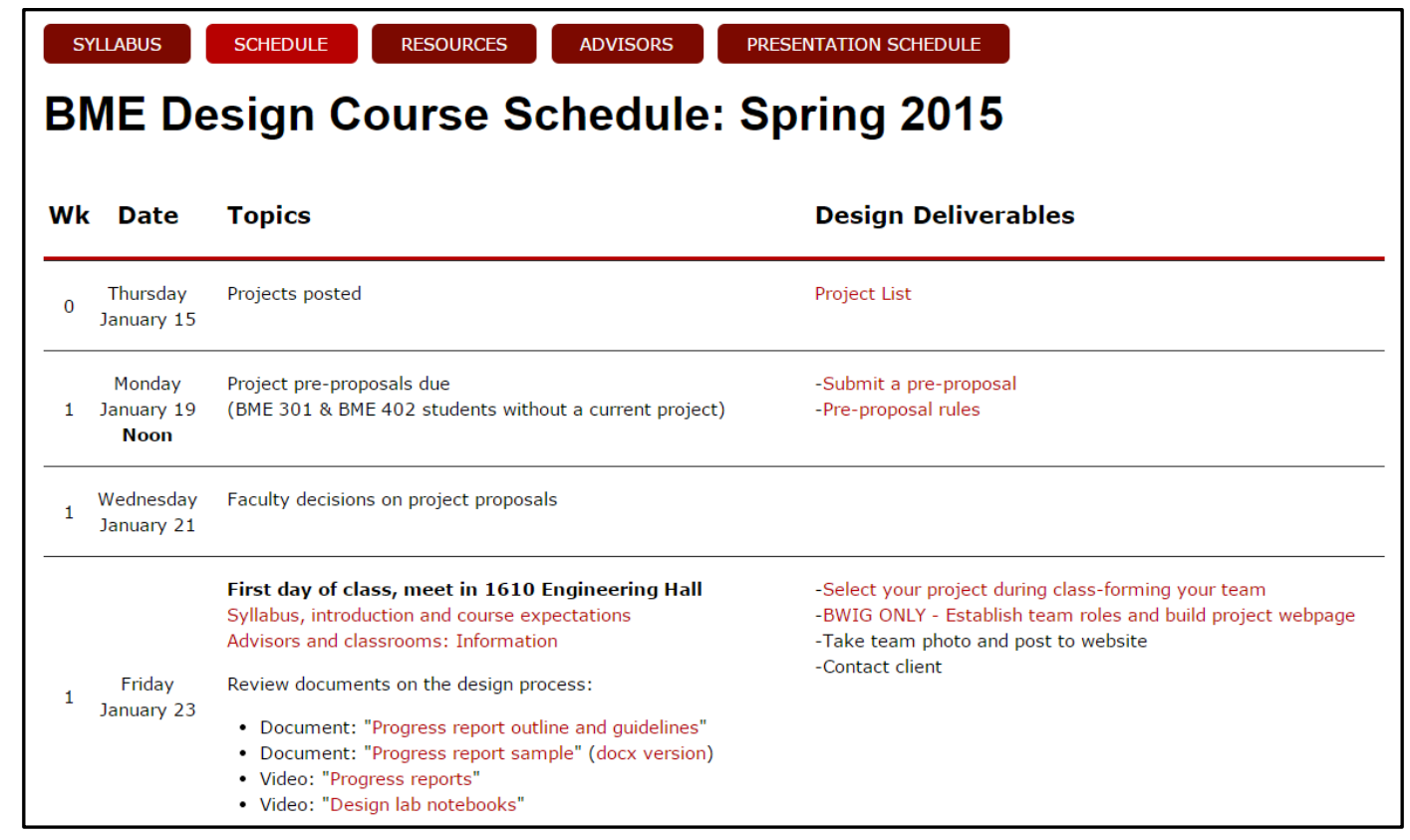

Fig. 4: Screenshot of the course schedule. The navigation bar at top of every course page contains links to the course syllabus, schedule, resources with pdf guidelines and links, advisor list with contact information and expertise, and the presentation schedule. 


\section{My Projects/Administration}

The entire Design course manager website is run through the 'My Projects' interface. Every contact that has been entered (student, client, advisor, and guests) can create an account with their email address and administer their account in My Projects. Since many of our users such as the clients, are not University affiliated, we choose to use this homegrown approach rather than using a University login. Personal information is encrypted within the database and all login and personal information is transmitted from the user's web browser to the server over an interface protected via Secure Socket Layer (SSL).

In My Projects, students, clients, and advisors have direct access to all projects they have been involved with. For advisors, this list is collapsible to display current projects and hide past projects to preserve screen space. Current students and advisors see the link to the course page. Advisors are also able to see the projects that have been submitted through the ideas interface (project pool, selection projects, and archives projects) as well as the pre-proposals submitted by students. Finally, advisors have access to a complete project lists of titles and members and contact information to easily access, copy, or click on their team's email addresses.

Administrators have additional access under My Projects to manage the various parts of the system. Administrators have the ability to view email lists in different configurations, allowing them to send email reminders to teams and certain team roles as necessary. The system also allows for the download of pre-formatted information for use in other University systems such as the creation of course evaluations and configuration of electronic design notebooks. ${ }^{5}$ User administration is also a key feature in this view - administrators can view and edit user information as necessary.

To aid in the configuration of the software for the semester, Administrators can use the 'Prepare for Semester' feature (as briefly mentioned above). This allows them to provide the BWIG Chair's contact information (this information is made available to students in Project Builder) and to specify the semester's dates. The system will then configure the database to prepare it for new projects, archive last semester's projects, and set up Selection to enable pre-selection proposals.

Finally, Administrators and advisors can access the 'Ideas and Selection Control Panel' from within 'My Projects'. The control panel is primarily used to manage new project submissions and for advisors to review projects and pre-proposals. Student information can be uploaded through this interface and desired team sizes specified for selection. Based on the desired team setup (i.e. 4 students per team) and the number of students enrolled in the course, the system will calculate the number of teams necessary to maximize team size. Finally, the course coordinator can also define the list of engineering specialties and skills that can be automatically applied to the various projects. During the Selection process, this interface is also used to access the student and advisor assignment and to view the classrooms associated with each project.

\section{Discussion}

Before the existence of our BME Design course manager as previously briefly described, all project idea solicitations were compiled manually for distribution to the students, then each name was written on a large Post it for selection. The students' names were printed and put in a hat for random draw, then called to physically walk up to the wall writing their name on the project they 
wanted. From the beginning of our design program, all projects had webpages and these were manually created by each team's BWIG with the framework being built by the BWIG Chair using a common style sheet. The overall time commitment from the course coordinator and the BWIG Chair was extensive even with only a hand-full of projects.

From what took over two hours with 25 projects during selection now only lasts thirty minutes with 52 projects. Additionally, there is minimal work on the front end, except the standard vetting of submitted project ideas, and no work on the back end. While, some students enjoyed creating the HTML and CSS for the webpage, others struggled and often did not upload documents. Now, the BWIG on each team has easy access to upload documents and update the project status and description all while meeting accessibility standards. One of the most significant advantages of using this system versus other CMS options is the ability of the clients (from outside of the University) to easily access the information at all times, and when appropriate, protect information that may contain intellectual property. Additionally, since up to 16 faculty might be teaching/advising design at one time, it also provides a means for the advisors to quickly find their team's information.

Other than regular bug fixes and release of new features, maintenance on the system is minimal. The software is built using HTML, CSS, PHP, and Javascript with a MySQL database storing the data running on a web server provided by our university. Most servers have the ability to run PHP and MySQL (the others run within a user's browser) and can be easily installed if the web server does not have this software preinstalled. By using these modern web technologies, our system has undergone several server software upgrades performed by our university's IT department and required no code changes to ensure continued functionality. Administrators and course coordinators do not need to know coding to change settings in the system as web interfaces provide the necessary options to change these settings, although it is helpful in order to be able to perform bug fixes and develop new features as necessary.

The system itself was built with maximum flexibility to meet potential future needs of the department or possibly be used by other departments. While the goal was to simplify and streamline the project selection and website creation part of the six design courses, it has taken on the role of collecting projects to providing resources for the students within the course.

\section{Conclusion}

As our department has been growing larger, our web course manager software has been evolving with the needs of the design course. The software has allowed our faculty to focus on the design process to ensure that students take the most away from the course. Through the five different areas of the software, we can individually manage and administer distinct portions of the course. Future work will aim to create a more seamless ability for the clients to request projects to continue within their My Projects, enhance the search feature to include engineering specialties, and potentially add the ability to enhance team dynamics by matching skills. ${ }^{4}$ Other design-based courses on our campus have express interest in using similar software and we are investigating making this software available to others. 


\section{References}

1. Tompkins, W.J.. "Implementing Design Throughout the Curriculum,” BMES, Chicago, Illinois,, 2006.

2. Tompkins, W. J., Beebe, D.J., Gimm, J. A., Nicosia, M. , Ramanujam, N., Thompson, P., Tyler, M. E., Webster, J. G.. "A design backbone for the biomedical engineering curriculum," Proc. of the 2nd Joint Conference of the IEEE Engineering in Medicine and Biology Society and the Biomedical Engineering Society, 2002, pp. 2595-2596.

3. Layton, R. A., Loughry, M. L., Ohland, M. W., \& Ricco, G. D. "Design and validation of a web-based system for assigning members to teams using instructor-specified criteria," Advances in Engineering Education, 2010; 2 (1), pp. 1-28.

4. Chesler, N.C., Brace, C.L., Tompkins, W.J. "Learning Assessment in a Design-Throughout the-Curriculum Program," American Society for Engineering Education Annual Conference, Vancouver, British Columbia, 2011 ; pp. 117.

5. Puccinelli, J.P., Nimunkar, A.J.. "An Experience with Electronic Laboratory Notebooks in Real-World, ClientBased BME Design Courses," American Society for Engineering Education Annual Conference, Indianapolis, 2014. 